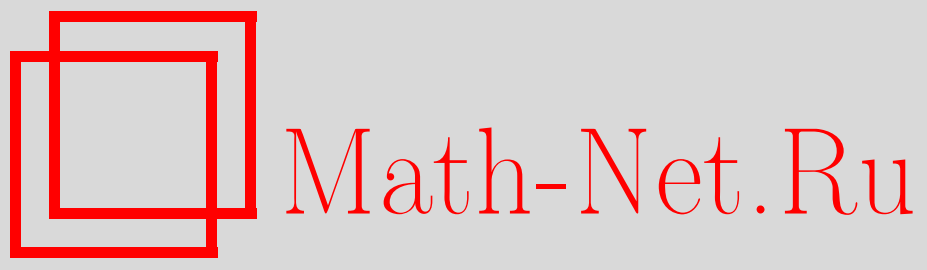

В. П. Маслов, Математические аспекты слабо неидеального бозе- и ферми-газа на кристаллической подложке, Функи. анализ и его прил., 2003, том 37, выпуск $2,16-27$

DOI: https://doi.org/10.4213/faa145

Использование Общероссийского математического портала Math-Net.Ru подразумевает, что вы прочитали и согласны с пользовательским соглашением

http://www . mathnet.ru/rus/agreement

Параметры загрузки:

IP : 54.80 .73 .141

26 апреля 2023 г., $17: 12: 53$

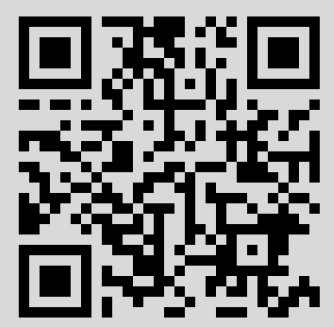


2003, т. 37, вып. 2, с. 16-27

УДК 517.9

\title{
Математические аспекты слабо неидеального бозе- и ферми-газа на кристаллической подложке
}

\author{
(c) 2003. В. П. МАСлов
}

В современном функциональном анализе часто рассматриваются уравнения с частными производными от бесконечного числа аргументов в бесконечной области. Вопрос об асимптотике решений уравнений при стремящемся к бесконечности числе аргументов, если одновременно стремится к бесконечности «объем» области, на которой рассматривается задача, близок к задачам квантовой статистики и термодинамики. Эти задачи в физике так же, как и в квантовой теории поля, часто рассматриваются на решетке (на разностной сетке). Это делается не только для некоторых упрощений и регуляризаций, но и потому, что такая постановка реализуется в кристаллических структурах.

С другой стороны, задачи квантовой статистики связаны с математической статистикой и теорией вероятностей. Известно, что квадрат модуля собственной функции уравнения Шрёдингера определяет плотность распределения частицы по координатам, отвечающую этому собственному значению, т. е. говоря на физическом языке, данному уровню энергии.

Однако, с какой вероятностью частицы распределены по уровням энергии уравнения Шрёдингера, в силу его линейности, казалось бы, в принципе, невозможно определить. В настоящей работе мы устраним это несоответствие, введя частотную вероятность для собственных значений, равную их кратности,

В самом начале прошлого века, в 1900 г., Паулем Друде и независимо от него Дж. Дж. Томсоном была предложена модель (теория Друде), согласно которой электрический ток в металлах переносится свободными электронами, поведение которых идентично поведению совокупности молекул идеального газа (концепция электронного газа или газа на «кристаллической подложке»). Эта теория объяснила огромный ряд экспериментальных закономерностей и фактов, позволила найти коэффициент электропроводности металлов и много новых эффектов.

С тех пор появилось много более точных теорий, однако экспериментаторы часто используют кристаллическую подложку, но при этом применяют формулы идеального бозе- и ферми-газа в пустоте, а в последнее время - формулы Боголюбова для слабо неидеального бозе-газа [1]. Работа Боголюбова, которая объяснила сверхтекучесть, открытую в конце 30-х годов, стала уже классической и является по существу чисто математической: в ней исследуется асимптотика симметрических относительно перестановки частиц решений многочастичного уравнения Шрёдингера на торе при числе частиц и радиусе тора, стремящихся к бесконечности так, чтобы отношение площади тора к числу частиц стремилось к константе.

В последних работах по наноэлектронике (ловушкам, дотам) большой интерес стал проявляться к следующей задаче: какие формулы применять для описания идеального газа в случае, когда число частиц газа конечно? При этом число 
частиц может быть не столь велико, как это принято считать [5]. Возможно, оно равно ста, а может быть, даже и меньше.

В данной работе рассмотрен несколько необычный идеальный газ. В обычном идеальном газе все частицы свободны. На самом деле существует более общее определение идеального газа - как совокупности частиц, находящихся в некотором поле, в некоторой общей потенциальной яме, но не взаимодействующих друг с другом [2]. Предъявленная в настоящей работе модель предоставляет возможность записывать формулы для конечного числа частиц идеального газ. Она как раз заключается в том, что каждая частица идеального газа не свободна, а именно находится на некоторой кристаллической подложке. Эти частицы не взаимодействуют друг с другом. Таким образом, получается модель идеального газа, но не находящегося в пустоте.

В настоящей работе предельный переход в термодинамику осуществляется не совсем обычным образом и не таким, как в цитируемой работе Боголюбова. Принято считать, что число частиц и отношение числа частиц к обычному объему стремятся к бесконечности. Мы же рассматриваем случай, когда отношение числа частиц к фазовому объему является конечной величиной. В традиционной термодинамике фазовый объем равен бесконечности. Во введенной модели свободная энергия и энтропия вычисляются в случае конечного числа частиц и конечного фазового объема. Получающиеся при этом формулы были уже известны [3]. Однако числа, которые в них входили, имели другой смысл. Эти формулы рассматривались как в квантовой механике, так и в теории вероятностей [4]. Но они не были получены из определенного гамильтониана, в котором берутся симметрические и антисимметрические решения, и не были связаны с рассматриваемой моделью.

Далее рассматривается сумма $N$ гамильтонианов, не взаимодействующих между собой. Полученные формулы не являются чем-то новым, а дают новую интерпретацию известных формул для идеального газа. В физической литературе эти два подхода к решению задач, первый - с помощью распределения частиц по ячейкам, второй - с помощью гамильтониана, действующего в симметрическом или антисимметрическом относительно перестановки частиц пространстве, не были состыкованы между собой. Каждая из задач решалась сама по себе. Получение формул распределения по ячейками из гамильтониана не было ни замечено, ни проведено.

Сначала рассмотрим простой пример, когда потенциал имеет вид ямы с двумя впадинами, симметричными относительно оси ординат (потенциал такого вида называют «ямой» Лифшица). Собственные функции в такой потенциальной «яме» симметричны относительно начала координат. Они либо четны, либо нечетны. Квадраты этих собственных функций симметричны относительно барьера, разделяющего потенциальные впадины. Если высоту барьера между этими двумя впадинами устремлять к бесконечности, то эта симметрия сохранится. И, следовательно, в пределе квадрат собственной функции, а значит и вероятности пребывания частицы в одной и в другой впадине будут одинаковыми.

Если мы устремим не барьер к бесконечности, а параметр $h$ (константу Планка) к нулю, т.е. будем рассматривать квазиклассический предел, то для собственных функций, собственные значения которых лежат ниже барьера энергии, в пределе переход будет запрещен. Тем не менее классическая частица будет как бы сидеть одновременно в двух впадинах, иначе говоря, как бы разделится на две части. 
Существует классическая теория, соответствующая такой модели. В ней можно представить «яму» Лифшица в виде двух чашек, соединенных между собой барьером, а частицу в виде маленькой дробинки. Если имеется какой-то внешний шум, например, молекулы бьются об эту дробинку, то очень редко дробинку этот шум будет перебрасывать из одной чашки в другую. Среднее время пребывания в той и другой чашке будет одинаковым. Пребывание частицы в левой и правой чашках будет равновероятно.

Это можно понимать и следующим образом. Допустим, в одной из впадин имеется квантовая частица, и при этом высота барьера конечна. Тогда со временем в силу резонанса частица может благодаря туннельному эффекту проникать через барьер, и в конце концов за большое время распределится равномерно в обеих впадинах. Если затем высоту барьера устремить к бесконечности, то можно рассмотреть два предела: один - по времени, другой - по высоте барьера. Эти два предела не перестановочны. Но если сначала устремлять к бесконечности время, а затем высоту барьера, то вероятность пребывания частицы и в той и другой впадине будет одинакова. При этом, если барьер высокий, то расстояние между собственными значениями находящихся в резонансе собственных функций будет очень мало. При высоте барьера, равной бесконечности, получится кратный (вырожденный) спектр.

Такая же ситуация возникает и в случае периодического потенциала. Допустим, сама система находится на окружности, и на этой окружности есть периодический потенциал, например, с $M$ впадинами. Тогда частица будет равновероятно сидеть во всех $M$ впадинах. Если при этом барьер между впадинами устремить к бесконечности, то частица все равно останется во всех этих впадинах с одинаковой вероятностью. Если считать, что собственная функция нормирована и вероятность пребывания в каждой впадине этого периодического потенциала есть интеграл от квадрата собственной функции по этой впадине, то этот интеграл будет равен $1 / M$. Если устремить барьеры между этими впадинами к бесконечности, то естественно, что это свойство симметрии в пределе сохранится (оно непрерывно зависит от высоты барьера). При этом каждое собственное значение будет иметь кратность $M$. Такая же ситуация, естественно, возникнет и в трехмерном случае, если имеется периодический по трем направлениям потенциал. Это означает наличие такого конечного кристалла, который удовлетворяет периодическим условиям Борна-Кармана, т. е. он как бы сам по себе замыкается на тор. На этом торе имеется периодическая решетка. Таким образом, в трехмерном случае, во-первых, получается, вообще говоря, существенно бо́льшая кратность и, во-вторых, она не обязательно одинаковая в разных впадинах, так как в трехмерном случае в каждой ямке могла быть еще и собственная кратность. Эта собственная кратность умножается на число впадин или, как говорят в случае кристаллов, на число ячеек кристалла.

Итак, рассмотрим именно такую модель, т. е. будем считать, что барьер между ячейками бесконечный и проникновение из одной ячейки в другую невозможно. Тем не менее частица будет сидеть во всех ячейках сразу. В рамках этой модели мы пренебрегаем расстоянием между точками спектра, определяемым туннельным эффектом. Эти собственные значения действительно очень близки, а именно, величина, пропорциональная расстоянию между ними, убывает экспоненциально с высотой барьера, и можно считать эти собственные значения одинаковыми. 
Пусть задан некоторый уровень энергии $E$. Рассмотрим число собственных значений или, иначе говоря, число уровней энергии, меньших либо равных энергии $E$, с учетом их кратностей. Это число будет равно числу таких уровней в одной ячейке, умноженному на число ячеек $M$. Теперь возьмем среднюю энергию этой системы. Для этого достаточно просто взять с точностью до константы сумму собственных значений, меньших либо равных $E$, с учетом их кратностей, иначе говоря, взять «шпур» данной системы относительно уровня энергии $E$. Он тоже равен сумме собственных значений, не превосходящих $E$, с учетом их кратностей, которые равны кратностям собственных значений одной ячейки, умноженной на число ячеек $M$. Отношение «шпура» к числу собственных значений есть внутренняя энергия этой системы. Так как при подсчете как средней, так и внутренней энергии, число ячеек $M$ и там, и там сокращается, то внутренняя энергия этой системы сводится к внутренней энергии одной ячейки. В классическом пределе внутренней энергией является фазовый интеграл по ямке, находящейся ниже уровня энергии $E$. Внутренняя энергия ячейки совпадает с фазовым объемом, который есть фазовый интеграл по энергиям, меньшим данной. Таким образом, если у нас в ячейке гамильтониан имеет вид $p^{2}+v(x)$, то фазовый объем совпадает с интегралом по области $p^{2}+v(x) \leqslant E$.

В силу сказанного выше мы рассмотрим самосопряженный оператор одной частицы $\widehat{H}$, имеющий $n$ различных собственных значений $\varepsilon_{1}, \ldots, \varepsilon_{n}$ с кратностями $G_{1}, \ldots, G_{n}$ соответственно, $\sum G_{i}=M$, и частотные вероятности $g_{i}=G_{i} / M$. Собственные функции гамильтониана $\widehat{H}$, отвечающие собственному значению $\varepsilon_{i}$, будем обозначать $\varphi_{\alpha_{i}}^{i}(\xi)$, где $\alpha_{i}=1, \ldots, G_{i}$. Будем считать, что переменная $\xi$ принимает конечное дискретное множество значений.

Рассмотрим идеальный газ из $N$ таких частиц, т. е. оператор $\widehat{H}_{N}=\sum_{1}^{n} \widehat{H}\left(\xi_{i}\right)$. Пусть $N_{i}$ - число частиц, отвечающих собственному значению $\varepsilon_{i}$, т. е. находящихся на энергетическом уровне $\varepsilon_{i}$. Гамильтониан $\widehat{H}_{N}$ действует на функции вида $\prod_{i=1}^{N} \varphi_{i}\left(\xi_{i}\right)$ следующим образом:

$$
\begin{aligned}
\widehat{H}_{N}\left(\varphi_{1}\left(\xi_{1}\right) \cdots \varphi_{N}\left(\xi_{N}\right)\right)=\widehat{H} & \left(\varphi_{1}\left(\xi_{1}\right)\right) \cdot \varphi_{2}\left(\xi_{2}\right) \cdots \varphi_{N}\left(\xi_{N}\right) \\
& +\varphi_{1}\left(\xi_{1}\right) \cdot \widehat{H}\left(\varphi_{2}\left(\xi_{2}\right)\right) \cdot \varphi_{3}\left(\xi_{3}\right) \cdots \varphi_{N}\left(\xi_{N}\right)+\ldots \\
& +\varphi_{1}\left(\xi_{1}\right) \cdots \varphi_{N-1}\left(\xi_{N-1}\right) \cdot \widehat{H}\left(\varphi_{N}\left(\xi_{N}\right)\right) .
\end{aligned}
$$

Таким образом, функции вида

$$
\Psi_{\{j, \alpha\}}\left(\xi_{1}, \ldots, \xi_{N}\right)=\prod_{s=1}^{N} \varphi_{\alpha_{j_{s}}}^{j_{s}}\left(\xi_{s}\right),
$$

где $\{j, \alpha\}$ обозначает набор $\left(j_{1}, \alpha_{j_{1}} ; j_{2}, \alpha_{j_{2}} ; \ldots ; j_{N}, \alpha_{j_{N}}\right)$, являются собственными функциями оператора Гамильтона $\widehat{H}_{N}$. Заметим, что среди индексов $j_{s}$, а также индексов $\alpha_{j_{s}}$ формулы (1) могут быть повторяющиеся.

Ясно, что $\sum_{i=1}^{n} N_{i}=N$. Собственным функциям (1) соответствует собственное значение

$$
\lambda_{\{N\}}=\sum_{i=1}^{n} N_{i} \varepsilon_{i},
$$

где $\{N\}-$ набор $\left\{N_{1}, \ldots, N_{n}\right\}$. 
Рассмотрим теперь симметричные собственные функции идеального газа из $N$ частиц.

Пространство симметричных (бозонных) собственных функций оператора $\widehat{H}_{N}$, отвечающих собственному значению $\lambda_{\{N\}}$, порождается функциями вида $\operatorname{Symm}_{\xi_{1}, \ldots, \xi_{N}} \Psi_{\{j, \alpha\}}\left(\xi_{1}, \ldots, \xi_{N}\right)$, где Symm - операция симметризации по $\xi_{i}$.

Для подсчета кратности собственного значения не важен порядок аргументов симметризуемой функции; поэтому можно рассматривать лишь те функции $\Psi_{\{j, \alpha\}}$, которые имеют вид

$$
\begin{array}{r}
\widetilde{\Psi}_{\{j, \alpha\}}\left(\xi_{1}, \ldots, \xi_{N}\right)=\varphi_{\alpha_{1}^{1}}^{1}\left(\xi_{1}\right) \cdots \varphi_{\alpha_{1}^{K_{1}}}^{1}\left(\xi_{K_{1}}\right) \varphi_{\alpha_{1}^{2}}^{2}\left(\xi_{K_{1}+1}\right) \cdots \varphi_{\alpha_{2}^{K_{2}}}^{2}\left(\xi_{K_{1}+K_{2}}\right) \\
\cdots \varphi_{\alpha_{n}^{1}}^{n}\left(\xi_{K_{1}+K_{2}+\cdots+K_{n-1}+1}\right) \cdots \varphi_{\alpha_{n}^{K_{n}}}^{n}\left(\xi_{N}\right) .
\end{array}
$$

В приведенном выше произведении могут стоять и одинаковые собственные функции оператора $\widehat{H}$. Рассмотрим произведение

$$
\varphi_{1}^{1}\left(\xi_{1}\right) \varphi_{1}^{1}\left(\xi_{2}\right) \cdots \varphi_{1}^{1}\left(\xi_{l_{1}}\right) \varphi_{2}^{1}\left(\xi_{l_{1}+1}\right) \cdots \varphi_{2}^{1}\left(\xi_{l_{1}+l_{2}}\right) \varphi_{3}^{1}\left(\xi_{l_{1}+l_{2}+1}\right) \cdots \varphi_{g_{1}}^{1}\left(\xi_{l_{1}+\cdots+l_{g_{1}}}\right),
$$

где $0 \leqslant l_{i} \leqslant N_{1}, \sum_{i=1}^{g_{1}} l_{i}=N_{1}$. Число таких всевозможных произведений равно числу способов распределения $N_{1}$ функций по $G_{1}$ классам. Это число есть число сочетаний

$$
C_{G_{1}+N_{1}+1}^{N_{1}}=\frac{\left(G_{1}+N_{1}-1\right) !}{N_{1} !\left(G_{1}-1\right) !} .
$$

Лемма 1. Кратность собственного значения $\lambda\{N\}$ симметричных собственных функций оператора Гамильтона $\widehat{H}_{N}$ равна

$$
\Gamma_{\left\{N_{i}\right\}\left\{G_{i}\right\}}=\prod_{i=1}^{n} \frac{\left(G_{i}+N_{i}-1\right) !}{N_{i} !\left(G_{i}-1\right) !},
$$

что по приведенному выше определению является частотной (ненормированной) вероятностью собственного значения $\sum_{1}^{n} N_{i} \varepsilon_{i}$ оператора $\widehat{H}_{N}$.

Определим, как обычно, свободную энергию рассмотренного идеального газа как

$$
\min _{N_{i}}\left\{\sum N_{i} \varepsilon_{i}-\theta \ln \Gamma_{\left\{N_{i}\right\}\left\{G_{i}\right\}}\right\},
$$

где $\theta-$ температура, а $S=\ln \Gamma_{\left\{N_{i}\right\}\left\{G_{i}\right\}}-$ энтропия. Тем самым мы получим распределение идеального газа на кристаллической подложке в случае конечного $N$.

Перейдем теперь к пределу $N \rightarrow \infty, M \rightarrow \infty, \lim (N / M)=\rho$, где $\infty>\rho \geqslant 0$, и применим формулу Стирлинга.

В этом случае, если определить частотные вероятности $N$ частиц как $N_{i} / N$, то для $p_{i}=\lim \left(N_{i} / N\right), g_{i}=\lim \left(G_{i} / M\right)$ мы получим следующее значение удельной энтропии:

$$
S=\sum_{i}\left(p_{i}+q_{i} \rho^{-1}\right) \ln \left(p_{i}+q_{i} \rho^{-1}\right)-p_{i} \ln p_{i}-q_{i} \rho^{-1} \ln q_{i} \rho^{-1} .
$$

Аналогично, удельная энтропия ферми-газа на кристаллической подложке равна

$$
S_{\mathrm{F}}=\sum_{i} q_{i} \rho^{-1} \ln q_{i} \rho^{-1}-p_{i} \ln p_{i}-\left(q_{i} \rho^{-1}-p_{i}\right) \ln \left(q_{i} \rho^{-1}-p_{i}\right) .
$$


Здесь $p_{i}$ и $q_{i}$ - вероятности: $\sum p_{i}=1, \sum q_{i}=1$. При $\rho \rightarrow 0$ они с точностью до константы $\ln \rho$ переходят в энтропию Шеннона $-p_{i} \ln \left(p_{i} / q_{i}\right)$. Этот предел показывает значение вероятностей $p_{i}$ и $q_{i}$ в теории информации [15].

В отличие от формулы (5) для свободной энергии, автор предлагает рассматривать оператор свободной энергии следующего вида $[6,7]$. Пусть $\delta\left(\lambda_{n}\right)$ есть кратность собственного значения $\lambda_{n}$ самосопряженного оператора $\widehat{A}=\int \lambda d E_{\lambda}$ (где $E_{\lambda}-$ спектральное семейство) с дискретным спектром в гильбертовом пространстве $H$. Оператор свободной энергии $\widehat{F}$ в этом же пространстве определяется формулой ${ }^{1)}$

$$
\widehat{F}=\int\{\lambda-\theta \ln \delta(\lambda)\} d E_{\lambda} .
$$

В нашем случае оператор $\widehat{A}$ есть оператор $\widehat{H}_{N}$, и минимальное собственное значение оператора $\widehat{F}$ совпадает с $(5)$, т. е. с обычным определением свободной энергии [3].

Аналогом минимального собственного значения для оператора Шрёдингера является его «нижний» уровень, «основное состояние», состояние, в которое в конце концов за большое время должна придти система (если маятник будет качаться долгое время, он в конце концов остановится).

Открытие сверхтекучести как бы опровергло этот постулат. При температуре, равной нулю, есть движение, энергия не равна нулю и это состояние сохраняется (как вычислено в последнее время, движение все-таки прекратится, но через миллионы лет).

В работе Н. Н. Боголюбова показано на примере многочастичного уравнения Шрёдингера, что при $N \rightarrow \infty$ существуют метастабильные собственные значения, которые должны «жить» достаточно долго и которые определяют тем самым состояние сверхтекучести. Это проделано при температуре, равной нулю.

Мы приведем здесь на основе изложенного выше модель оператора свободной энергии, которая показывает, что у последнего тоже могут существовать метастабильные собственные значения.

Мы будем рассматривать, как это обычно делается в физической теории металлов, только две «зоны», в нашем случае - два собственных значения одночастичного гамильтониана $\varepsilon_{1}$ и $\varepsilon_{2}$. Взаимодействие также будет выбрано так, чтобы оно отвечало физической задаче [11].

В квантовой механике, как уже говорилось, квадрат модуля собственной функции является плотностью вероятности пребывания частицы в координатной точкe.

Поскольку мы рассматриваем распределения по собственным значениям (уровням энергии), то важно знать вероятности перехода с одного уровня на другой.

Пусть имеется $N$ частиц вида

$$
\begin{aligned}
V_{N}^{(s)}=\sum H_{i}+\frac{1}{N} \sum \sum H_{i_{1}, i_{2}} \\
\quad+\frac{1}{N^{3}} \sum \sum \sum H_{i_{1}, i_{2}, i_{3}}+\cdots+\frac{1}{N^{s}} \sum \ldots \sum H_{i_{1}, \ldots, i_{s}},
\end{aligned}
$$

1)На моем докладе на математическом обществе в 2000 г. В. И. Арнольд спросил меня, как определить оператор свободной энергии в случае непрерывного спектра. Эта проблема решена в [6]. 
где $s$ сколь угодно велико, но много меньше $N$, а $H_{i_{1}, \ldots, i_{k}}$ - оператор, действующий только на $k$ частиц, например,

$$
H_{i_{1} i_{2}} f\left(x_{1}, \ldots, x_{N}\right)=H\left(x_{i}, x_{j}\right) f\left(x_{1}, \ldots, x_{N}\right),
$$

т. е. на всех координатах, кроме $x_{i_{1}}$ и $x_{i_{2}}$, он действует как единичный оператор.

Вероятностью перехода частиць из состояния $\lambda_{k}$ (отвечающего собственному значению $\lambda_{k}$ оператора $H_{N}$ ) в состояние $\lambda_{k^{\prime}}$ (отвечающее собственному значению $\lambda_{k^{\prime}}$ ) под действием оператора $V_{N}$ называется (в квантовой механике) квадрат модуля скалярного произведения $\left(\Psi_{\lambda_{k}}, V_{N} \Psi_{\lambda_{k^{\prime}}}\right)$.

Это выражение называется матричным әлементом. Это важное физическое понятие существенно меняет представление о стандартных понятиях спектра оператора в функциональном анализе, разбивая спектр оператора на спектральные серии $[6,10]$.

В физике имеет место закон, который Боголюбов называет «энергетически выгодное состояние». Частица с течением времени теряет энергию и переходит на более низкий уровень под влиянием возмущений типа «трения». Сразу из «состояния» уровня $\lambda_{i}$ на уровень $\lambda_{i-k}$ она перейти не может, однако она может сначала перейти на уровень $\lambda_{i-1}$, затем на уровень $\lambda_{i-2}$ и т. д., пока не спустится на уровень $\lambda_{i-k}$, а затем в конце концов на нижний уровень $\lambda_{1}$, который и называется в физике «основным состоянием».

Для того чтобы описать математически возмущения типа трения, мы должны все матричные элементы, отвечающие переходу на более высокие уровни энергии (на бо́льшие собственные значения), положить равными нулю. Такой оператор возмущения $\widetilde{V}_{N}^{(s)}$ не будет уже самосопряженным.

Мы рассмотрим следующую простую модель: каждые две частицы, чтобы попасть на более высокую зону, отдают квант энергии, равный $\nu / N$, где

$$
2\left(\varepsilon_{2}-\varepsilon_{1}\right)>\nu>\varepsilon_{2}-\varepsilon_{1}, \quad \varepsilon_{2}>\varepsilon_{1} .
$$

Пусть $\mathscr{L}$ - пространство функций $\Psi\left(x_{1}, \ldots, x_{N}\right)$, симметричных (антисимметричных) относительно дискретных переменных ${ }^{1)} x_{i}, i=1, \ldots, N$, которые принимают значения $1 / M, 2 / M, \ldots,(M-1) / M, 1$. Гамильтониан, отвечающий данной математической задаче, действует на функции пространства $\mathscr{L}$ следующим образом:

$$
\widehat{H}_{N} \Psi\left(x_{1}, \ldots, x_{N}\right)=\left(\sum_{i=1}^{N} \lambda\left(x_{i}\right)+\frac{1}{N} \sum_{i=1}^{N} \sum_{j=i+1}^{N} V\left(x_{i}, x_{j}\right)\right) \Psi\left(x_{1}, \ldots, x_{N}\right),
$$

где $\lambda(x)=\varepsilon_{1}$ при $1 / M \leqslant x \leqslant G_{1} / M$ и $\lambda(x)=\varepsilon_{2}$ при $\left(G_{1}+1\right) / M \leqslant x \leqslant 1$; кроме того, $V\left(x, x^{\prime}\right)=-V$, если $\left(G_{1}+1\right) / M \leqslant x \leqslant 1$ и $\left(G_{1}+1\right) / M \leqslant x^{\prime} \leqslant 1$; во всех остальных случаях $V\left(x, x^{\prime}\right)=0$.

Введем функции $\varphi_{i}(x), i=1, \ldots, G_{1}$, и $\phi_{j}(x), j=1, \ldots, G_{2}$, где $G_{2}=M-G_{1}$, следующим образом:

$$
\varphi_{i}(x)=\left\{\begin{array}{ll}
1 & \text { при } x=i / M, \\
0 & \text { при } x \neq i / M,
\end{array} \quad \phi_{j}(x)= \begin{cases}1 & \text { при } x=\left(j+G_{1}\right) / M, \\
0 & \text { при } x \neq\left(j+G_{1}\right) / M .\end{cases}\right.
$$

1) С физической точки зрения задача рассматривается в $p$-представлении, и следовало бы, согласно физическим обозначениям, писать вместо координаты $x_{i}$ импульс $p_{i}$. 
Собственные функции гамильтониана (9) будем нумеровать двумя наборами неотрицательных целых чисел, $\{k\}=k_{1}, \ldots, k_{G_{1}}$ и $\{l\}=l_{1}, \ldots, l_{G_{2}}$, такими, что

$$
\sum_{i=1}^{G_{1}} k_{i}+\sum_{j=1}^{G_{2}} l_{j}=N .
$$

Собственная функция $\Psi_{\{k\},\{l\}}$ выражается через функции (10) следующим образом:

$$
\begin{aligned}
& \Psi_{\{k\},\{l\}}=\underset{x_{1}, \ldots, x_{N}}{\operatorname{Symm}}\left(\varphi_{1}\left(x_{1}\right) \cdots \varphi_{1}\left(x_{k_{1}}\right) \varphi_{2}\left(x_{k_{1}+1}\right) \cdots \varphi_{2}\left(x_{k_{1}+k_{2}}\right) \cdots \varphi_{G_{1}}\left(x_{N_{1}}\right)\right. \\
& \left.\quad \times \phi_{1}\left(x_{N_{1}+1}\right) \cdots \phi_{1}\left(x_{N_{1}+l_{1}}\right) \phi_{2}\left(x_{N_{1}+k_{1}+1}\right) \cdots \phi_{2}\left(x_{N_{1}+k_{1}+k_{2}}\right) \cdots \phi_{G_{2}}\left(x_{N}\right)\right),
\end{aligned}
$$

где $\operatorname{Symm}_{x_{1}, \ldots, x_{N}}$ - оператор симметризации по переменным $x_{1}, \ldots, x_{N}$, а $N_{1}$ зависит от набора $\{k\}$ следующим образом:

$$
N_{1}=\sum_{i=1}^{G_{1}} k_{i} .
$$

Для случая ферми-газа мы рассматриваем оператор антисимметризации Asymm вместо Symm. Далее также будем использовать обозначение $N_{2}=N-N_{1}$. Собственное значение гамильтониана $\widehat{H}_{N}$, отвечающее собственной функции (11), имеет вид

$$
\lambda\left(N_{2}\right)=\varepsilon_{1} N+\left(\varepsilon_{2}-\varepsilon_{1}\right) N_{2}-\frac{V N_{2}\left(N_{2}-1\right)}{2 N},
$$

кратность этого собственного значения для симметрического случая (бозе-газа) равна

$$
\Gamma_{\mathrm{B}}\left(N_{2}\right)=\frac{\left(N-N_{2}+G_{1}-1\right) !}{\left(N-N_{2}\right) !\left(G_{1}-1\right) !} \frac{\left(N_{2}+G_{2}-1\right) !}{N_{2} !\left(G_{2}-1\right) !} .
$$

Для антисимметрического случая (ферми-газа) она равна

$$
\Gamma_{\mathrm{F}}\left(N_{2}\right)=\frac{G_{1} !}{\left(N-N_{2}\right) !\left(G_{1}-N+N_{2}\right) !} \frac{G_{2} !}{N_{2} !\left(G_{2}-N_{2}\right) !} .
$$

Исследуем собственные значения (12). Из их вида следует, что выполняются следующие неравенства:

$$
\begin{array}{ll}
\lambda\left(N_{2}+1\right)>\lambda\left(N_{2}\right) & \text { при } N_{2} \leqslant K-1, \\
\lambda\left(N_{2}+1\right)<\lambda\left(N_{2}\right) & \text { при } \quad N_{2} \geqslant K,
\end{array}
$$

где $K$ имеет вид

$$
K=\frac{\varepsilon_{2}-\varepsilon_{1}}{V} N
$$

Из (15) следует, что собственное значение $\lambda(0)$ является наименьшим из всех (глобальный минимум) собственных значений. Кроме того, из этих неравенств следует, что собственное значение $\lambda(N)$ является локальным минимумом собственных значений, так как для любого $N_{2}$, такого, что $K \leqslant N_{2}<N$, справедливо неравенство $\lambda\left(N_{2}\right)<\lambda(N)$. 
Рассмотрим возмущения гамильтониана $\widehat{H}_{N}$ самосопряженными операторами $H_{s}$, которые действуют в пространстве $\mathscr{L}$ как операторы умножения на функции

$$
H_{s}\left(x_{1}, \ldots, x_{2}\right)=\sum_{1 \leqslant i_{1} \neq i_{2} \neq \cdots \neq i_{s} \leqslant N} U_{s}\left(x_{i_{1}}, x_{i_{2}}, \ldots, x_{i_{p}}\right),
$$

где $s \geqslant 1$, а функции $U_{s}$ действительные. Такие операторы $H_{s}$ являются операторами $s$-частичного взаимодействия. Легко убедиться, что для матричных элементов таких операторов по собственным функциям (11) имеет место равенство

$$
\left(\Psi_{\{k\},\{l\}}, H_{s} \Psi_{\left\{k^{\prime}\right\},\left\{l^{\prime}\right\}}\right)=0 \quad \text { при } \sum_{i=1}^{G_{1}}\left|k_{i}-k_{i}^{\prime}\right|+\sum_{j=1}^{G_{2}}\left|l_{j}-l_{j}^{\prime}\right|>2 s .
$$

Поскольку $s=O(1)$, то из (18) и (15) вытекает следующее утверждение.

Под действием s-частичных возмущений собственные функиии, отвечающие глобальному и локальному минимумам собственных значений, могут переходить только в состояния, у которых собственные значения больше.

Согласно концепции Л. Д. Ландау, это означает, что состояние локального минимума является сверхтекучим.

Если перейти к другому «удельному» масштабу, то

$$
\lambda_{p}=\frac{\lambda\left(N_{2}\right)}{N}=\varepsilon_{1}(1-p)+\varepsilon_{2} p-\nu \frac{p^{2}}{2}-\frac{p}{2 N}, \quad p=\frac{N_{2}}{N}, 0 \leqslant p \leqslant 1 .
$$

Последний член стремится к нулю при $N \rightarrow \infty$; поэтому мы им будем пренебрегать. Спектр в этом пределе оказывается непрерывным.

Основным состоянием (глобальным минимумом) является $\bar{\lambda}_{0}=\varepsilon_{1}$. Максимальным является $\lambda_{p}$ при $p=\left(\varepsilon_{2}-\varepsilon_{1}\right) / \nu$. Имеется также и второе минимальное значение $\lambda_{1}$ при $p=1$, равное $\varepsilon_{2}-\nu / 2$.

Если применить к данной задаче возмущение трения $\widetilde{V}_{s}$, то, зная собственные функции (3), легко показать, что часть собственных значений (частиц, находящихся на высоких уровнях энергии) будет под влиянием возмущения трения постепенно переходить через ближайшее собственное значение на абсолютный минимум $\lambda_{0}$, а часть - на локальный минимум $\lambda_{1}$. Переход же с $\lambda_{1}$ на $\lambda_{0}$ асимптотически (при $N \gg 1$ ) запрещен. Поэтому возможно достаточно долгое нахождение на уровне $\lambda_{1}>\lambda_{0}$. На физическом языке об уровне $\lambda_{1}$ говорят, что это метастабильное состояние. Если $\lambda_{0}$ отвечает нулевой энергии, то $\lambda_{1}-$ уже ненулевой, а частицы, обладающие ненулевой энергией (кинетической), движутся с некоторой скоростью.

Итак, предположим теперь, что возмущение гамильтониана $\widehat{H}_{N}$ является $s$-частичным несамосопряженным оператором (трения) $\widetilde{V}_{s}$ и допускает только переходы на уровни с меньшими собственными значениями. В таком случае из (18) и неравенства (15) следует, что среди собственных функций (11) выделяются два класса. Собственные функции $\Psi_{\{k\},\{l\}}$, для которых справедливо неравенство

$$
N_{2}=\sum_{j=1}^{G_{2}} l_{j} \leqslant N \lambda_{p_{1}}-s-1,
$$


под действием указанного возмущения со временем придут в состояние глобального минимума, а собственные функции, для которых выполняется неравенство

$$
N_{2}=\sum_{j=1}^{G_{2}} l_{j} \geqslant N \lambda_{p_{1}}+s,
$$

m.е. $N_{2} / N>\lambda_{p_{1}}+O(1 / N)$, под действием указанного возмущения со временем придут в состояние локального минимума.

Пусть $\lambda\left(N_{2}, G_{1}, G_{2}\right)$ - собственные значения оператора свободной энергии $\widehat{F}$, a $\Lambda(p, q)=\lambda / N$,

$$
\Lambda_{F}=\varepsilon_{1}(1-p)+\varepsilon_{2} p-\nu \frac{p^{2}}{2}-\theta \ln \Gamma_{\mathrm{B}}, \quad 0 \leqslant p \leqslant 1 .
$$

В ферми-газе $\ln \Gamma_{\text {В }}$ заменяется на $\ln \Gamma_{\mathrm{F}}$. Критические значения $\Lambda_{F}$, определяемые из уравнения $\partial \Lambda_{F} / \partial p=0$, при достаточно малых $\theta$ имеют три значения: первое - минимум, второе - локальный минимум и третье - максимум:

$$
\begin{gathered}
p_{1} \approx \frac{q_{2}}{\rho+1-q_{2}} \exp \left(-\frac{\varepsilon}{\theta}\right), \quad p_{2} \approx 1-\frac{1-q_{2}}{\rho+q_{2}} \exp \left(-\frac{\nu-\varepsilon}{\theta}\right), \\
p_{3} \approx \frac{\varepsilon}{\nu}+\frac{\theta}{\nu} \ln \frac{\varepsilon}{\nu-\varepsilon} \frac{\nu\left(1-q_{2}\right)+\rho(\nu-\varepsilon)}{\nu q_{2}}, \quad \varepsilon=\varepsilon_{2}-\varepsilon_{1}
\end{gathered}
$$

(для бозе-газа).

Собственные функции оператора свободной энергии совпадают с собственными функциями гамильтониана $H_{N}$. Рассмотрим собственные значения оператора свободной энергии $\widehat{F}$ и тот же оператор трения $\widetilde{V}_{s}$, т. е. оператор, матричные элементы которого по собственным функциям (3) при переходе от нижних собственных значений оператора свободной энергии к более высоким равны нулю (см. выше). Тогда все собственные значения оператора свободной энергии при $p>p_{3}$ будут в результате многократного применения оператора трения стремиться к локальному минимуму - метастабильному сверхтекучему состоянию свободной энергии.

Состояние $p_{2}$ обладает также свойством локальной устойчивости (метастабильности), т. е. при возмущении оператором $\alpha H_{s}$, где $\alpha$ достаточно мало, значение $\lambda_{F}$ может лишь увеличиваться.

Критические значения температуры, при которой происходит фазовый переход, определяются из уравнений $\partial f / \partial p=0$ и $\partial^{2} f / \partial p^{2}=0$.

Критическая точка возникает там, где пересекаются две кривые $S_{1}(\theta)$ и $S_{2}(\theta)$, энтропии, отвечающие максимальной точке и точке локального минимума. В этой точке $\theta_{c}$ электроемкость $\partial S / \partial \theta$ обращается слева в $\infty$, как $\left(\theta-\theta_{c}\right)_{+}^{-1 / 2}$. Это означает на физическом языке, что имеет место фазовый переход с критическим индексом $1 / 2$. Отметим, что кривая $\theta(S)$ гладкая и совершает в критической точке поворот, где $S$ играет роль импульса, а $\theta-$ координаты (см. [8]). Так как точка поворота простая, то, согласно [8], применяя туннельный канонический оператор, который в этом случае превращается в функцию Эйри, получаем аналогично теореме 2 [8] следующее утверждение: 
Все приближения при $N \rightarrow \infty$ имеют точность $O(1 / N)$ за исключением окрестности критической точки. В критической точке из $p_{2}$ вычитается величина

$$
\frac{1}{6} \frac{\ln N}{N} .
$$

После прохождения критической точки метастабильность (сверхтекучесть) пропадает, начинается торможение и в конце концов под влиянием оператора трения частицы попадают на глобальный минимум, т. е. останавливаются.

Таким образом, из нашей модели получается, что если нагревать метастабильное (сверхтекучее) состояние, то при температуре $\theta_{0}$ совершается переход в «нормальное» состояние (состояние с трением). Это явление не есть обычный фазовый переход, когда имеет место скачок энтропии или электропроводности. Это есть «перескок» с одной термодинамики, определяемой одними термодинамическими потенциалами [3], на другую, определяемую другими термодинамическими потенциалами. Если трение очень велико, как это бывает в узком капилляре, то произойдет внезапная остановка сверхтекучей жидкости. Этот эффект, открытый в 1938 г. Д. Алленом и Х. Джонсом, называется эффектом фонтанирования [11], поскольку при такой внезапной пробке из крохотной дырочки над точкой нагревания начинает бить фонтан (высота фонтана для трубки $10^{-4}$ см достигала 14 см). Эта внезапная закупорка капилляра, приводящая к эффекту «фонтана», каскада, срыва, имеет место и в экономике, в биматричной игре, когда налог превышает некоторую критическую величину [13]. Этот эффект называется «пробоем» цены акций.

Мы, таким образом, получаем как бы две термодинамики: обычную, т. е. отвечающую минимальному значению свободной энергии, и другую термодинамику, отвечающую состоянию $p_{2}$. Эта вторая термодинамика при температуре, равной нулю, совпадает с метастабильным уровнем энергии многочастичного уравнения Шрёдингера, который, согласно общепризнанной концепции Боголюбова-Ландау, описывает сверхтекучее состояние. Таким образом, сверхтекучее состояние трактуется не как гидродинамическое движение жидкости, а как равновесное термодинамическое явление.

До работы Боголюбова Тиссой и Ландау была построена феноменологическая полугидродинамическая двухжидкостная модель, рассматривающая жидкость, состоящую из смеси двух компонент: нормальной и сверхтекучей, в частности, при температуре, равной нулю. Боголюбов показал, что явление сверхтекучести связано с уравнением Шрёдингера и является некоторым равновесным состоянием. Модель Тиссы-Ландау объясняла на том же феноменологическом уровне и указанный эффект фонтанирования.

«Вторая» термодинамика, построенная в настоящей работе для сверхтекучего состояния, объясняет эффект фонтанирования при достаточно большом трении (частом действии оператора трения). Если же трение не столь велико, например, труба, по которой «течет» сверхтекучая жидкость, достаточно большого радиуса (т.е. оператор трения действует не столь часто), то при $\theta>\theta_{c}$ жидкость будет продолжать течь как обычная вязкая жидкость, т. е. термодинамика сверхтекучей жидкости перейдет в гидродинамику обычной жидкости.

Таким образом, действие оператора трения должно быть связано с конкретной постановкой краевой задачи. Эта проблема выходит за рамки равновесной термодинамики. 


\section{ЛИТЕРАТУРА}

1. Боголюбов Н. Н. Избранные труды. Т. 2, Киев, 1970.

2. Дирак П. А. Принципы квантовой механики. М., 1960.

3. Ландау Л. Д., Лифшиц Е. М. Статистическая физика. Наука, М., 1976.

4. Ширяев А. Н. Вероятность. Наука, М., 1989.

5. Алексеев B. A. Функции распределения числа частиц в конденсате захваченного в ловушку идеального бозе-газа. ЖЭТФ, 119, вып. 4, 700-709 (2001).

6. Маслов В. П. Квантование термодинамики и ультравторичное квантование. Институт компьютерных исследований, М., 2001.

7. Maslov V. P. Spectral Series and Quantization of Thermodynamics. RJMP, 9, No. 1, 112-122 (2002).

8. Маслов В. П. Аналитическое продолжение асимптотических формул и аксиоматика термодинамики и квазитермодинамики. Функц. анализ и его прил., 28, вып. 4, 28-41 (1994).

9. Ландау Л. Д. Квантовая механика. Часть 1. Гостехиздат, М., 1966.

10. Maslov V. P. Quasi-Particles Associated with Lagrangian Manifolds Corresponding to Semiclassical Self-Consistent Fields. IV-XI. Russian J. Math. Phys., 3, No. 3, 401-406, No. 4, 529-534 (1995), 4, No. 1, 117-122, No. 2, 265-270, No. 4, 539-546 (1996), 5, No. 1, 123-130, No. 2, 273-278, No. 3, 405-412 (1997).

11. Маслов В. П. Модель слабо неидеального бозе-газа. Фазовый переход в сверхтекучем состоянии и эффект фонтанирования. Вестник МГУ, серия «Физика. Астрономия», № 1, 3-11 (2003).

12. Маслов В. П. Двухуровневая модель слабо неидеального бозе-газа. Фазовый переход в метастабильном (сверхтекучем) состоянии. ДАН, 389, № 4, 1-2 (2003).

13. Маслов В. П. Фазовые переходы в вероятностной игре. Матем. заметки, 73, № 4, 637-640 (2003).

14. Маслов В. П., Шведов О. Ю. Метод комплексного ростка. Эдиториал УРСС, М., 2000.

15. Маслов В. П. Замечание о компьютерно-ориентированном языке. Пробл. передачи информации, 39, вып. 3 (2003) (в печати).

16. Кеезом В. Гелий. Пер. с англ. с доп. Е. М. Лифшица и Э. Л. Андроникашвили, М., 1949.

17. Физика низких температур. Пер. с англ.. М., 1959, гл. Х.

Московский государственный университет им. М. В. Ломоносова, физический факультет

Поступило в редакцию 13 марта 2003 г. 